\title{
A comparison of methodological frameworks for digital learning game design
}

\author{
Alysson Diniz dos Santos and Piero Fraternali \\ Universidade Federal do Ceara, Instituto Universidade Virtual \\ Av. Humberto Monte, s/n, bloco 901, 60.440-554, Fortaleza, Brazil \\ alysson@virtual.ufc.br \\ Politecnico di Milano, DEIB \\ Via Ponzio 34/5, 20133, Milano, Italy \\ piero.fraternali@polimi.it
}

\begin{abstract}
Methodological frameworks guide the design of digital learning game based on well founded learning theories and instructional strategies. This study presents a comparison of five methodological frameworks for digital learning game design, highlighting their similarities and differences. The objective is to support the choice of an adequate framework, aiming to promote them as a way to foster principled digital learning games design. This paper concludes that: (i) interactivity, engagement and increasing complexity of challenges are fundamental factors to digital learning game design; (ii) the pedagogical base, the target, the possibility of doing game assessment and the presence of practical guidelines are the selection criteria that influence most the choice of a methodological framework, and (iii) the development of digital learning games - preferably by different research teams - is needed to provide empirical evidence of the utility of framework-based design.
\end{abstract}

Keywords: digital learning, educational, games, game-based learning, methodological frameworks

\section{Introduction}

Although digital learning games (DLGs) are well established as a research field [1], strong scientific evidence of the claimed benefits - e.g. higher user motivation, better selective attention and improvements on analytical, spatial and psycho-motor skills, among others [2] - is still required to support DLGs design, development and usage [1]. The use of established learning theories and instructional strategies on the design of the DLGs is fundamental to enhance gamebased learning [3]. With games grounded on learning theories and instructional strategies, researchers would be able to manipulate key variables and determine which factors have the greatest effect on learner motivation and achievement [3].

Recently, researchers proposed methodological frameworks to guide DLGs design coupling learning theories and instructional strategies with traditional game 
design aspects [4-8]. This paper presents a further look on these frameworks, analyzing their similarities, highlighting their differences, and investigating to which extent they were tested and used. Such a study may help the selection for a framework to base a DLG design, give insights on framework development and application to DLG design and, ultimately, contribute to improving DLGs effectiveness, through the use of adequate design methods. Specifically, the objectives of this work are:

- to identify and illustrate the most prominent frameworks for DLG design;

- to establish a comparison among them, highlighting their similarities and differences and,

- to expose open problems and research opportunities for both framework and DLGs designers.

Previous studies [3, 9-11] examined the pedagogical foundations that grounded DLGs design. Nevertheless, none of these cite any methodological framework directly. Ibrahim and Jaafar (2009) [5] discuss four methodological frameworks that influenced the conception of their own model, the Educational Game Design Framework (described in Section 6). However, the authors did not establish any comparison among the presented frameworks, using them just to support the proposal of their own approach.

The following Sections from 2 to 6 describe each analyzed methodological framework. Section 7 presents a comparison of the analyzed approaches, Section 8 discusses open problems and research directions, and Section 9 summarizes the findings of the paper.

\section{RETAIN Model}

The Relevance Embedding Transfer Adaptation Immersion \& Naturalization (RETAIN) model is founded on instructional methods and learning theories that are closely aligned with modern game design principles [7]. The RETAIN model is based on six mains aspects: relevance, embedding, transfer, adaptation, immersion and naturalization.

Relevance addresses three different aspects: (i) the learning materials should be relevant to learners, their needs and learning style; (ii) the instructional units should be relevant to one another, i.e., instructional units should be introduced and set in context with previously learned materials; and (iii) the game has to be relevant to reality, which includes insights on how to use the fantasy, i.e. the fiction supported by the narrative, commonly present in games. A related aspect refers to appropriately embedding content into the game fantasy. The intent is to integrate the educational content in such a way as to make it intrinsic to the fantasy context of the game. Learning and gameplay should function together seamlessly [7].

Knowledge transfer and adaptation are tightly related. The first aspect refers to the ability to teach player-learners how to transfer knowledge from one situation to another, and can be achieved through recall stimulation. The second 
refers to knowledge acquisition and can be achieved through assimilation - interpreting events in terms of previous known ones - and accommodation - alteration or creation of new knowledge, expanding the player understanding [7].

Immersion is the creation of a belief in the enveloping fantasy of the digital environment. It can be measured hierarchically from a simple interaction/reaction to being fully engaged to the context of the game. Adequate interactivity and a high level of engagement (provided by well-designed games) favor immersion [7].

Naturalization refers to automaticity or spontaneous knowledge, in which a learner uses the learned information habitually and consistently, monitors it, but does not have to devote significant mental resources to think about it. Games that are re-playable, i.e. the player enjoys to play repeated times, stimulate naturalization [7].

To simplify the framework use, the authors defined a table that classifies each of the five presented aspects in four levels (from 0 to 3 ). Each level has its set of requirements for the game to be considered at that level in a specific aspect. In a typical example, a game would be: level 1 in relevance, level 2 in embedding, level 2 in transfer, level 0 in adaptation, level 3 in immersion, and level 2 in naturalization. In addition, the authors classified the importance of each aspect, by the definition of a weighting scale. The table coupled with the weighting scale can be used to orient the development of a DLG and to evaluate the effectiveness of an already-developed one. No game so far declared to use RETAIN in its design.

\section{The 'I's Framework}

The 'I's framework [8] is based on a constructivist point of view, i.e. the players should learn by constructing new knowledge, connecting a new to a prior experience. The framework consists of a hierarchy of six elements, organized from low to high importance: identity, immersion, interactivity, increasing complexity, informed teaching, instructional.

Identity refers to the ability of capturing player's attention and tricking him into believing he is a unique individual within the environment - through a selectable avatar, for example. With a strong sense of identity and presence a player later can easily feel immersed and emotionally engaged with the game. Immersion is about having a heightened sense of presence in the environment, being engaged with the content and thus intrinsically motivated to succeed in the challenge of the game. The author argues that through high interactivity, adequate challenge level, appropriate feedback and user interface a game can harness immersion [8].

Adequate increasing complexity enhances the education provided by the game. Game challenges should fit the player increasing ability, aiming at a pleasurable frustration state - in which the player feels stimulated to try harder when facing a defeat. A DLG has to provide good level design and reward system to support adequate increasing complexity. Regarding feedback, informed teaching approaches embedded assessments within DLGs. A DLG can use in-game data 
(server-side data, ID, time, location, patterns of use and interaction, chat-logs and other tools) to run a posteriori analysis on players' proficiency [8].

Being instructional is the aim of any DLG. To achieve it, a DLG should present the previous elements - identity, immersion, interactivity, increasing complexity and informed teaching. Furthermore, other aspects can enhance the instructional power of the DLG. It should be adequately integrated to the curriculum, being re-playable and connected to traditional lab activities. The teacher should be responsible for creating scaffold-structuring interactions and developing instruction in small steps, based on tasks the learner is already capable of performing independently.

The author presents a game concept, which exemplify the DLG design process. However, no game so far reported to use the 'I's framework in its design.

\section{Game Object Model II}

The Game Object Model II (GOM-II) relates pedagogical dimensions of learning with game elements, based on the object oriented conceptual design paradigm [4]. The framework is focused on the development of adventure educational games and is organized around five core concepts: definition, narrative, gender, social collaboration and challenges-puzzles-quests:

The definition of a DLG refers to its learning potential. A well defined DLG should: (i) require the player to learn new strategies and skills and solve evermore complex challenges or puzzles; (ii) identify and exploit complex relationships between simulated and real characters, and (iii) solve ethical dilemmas [4].

The narrative defines the fantasy of the game. A good narrative should allow players to actively construct their own meaning/understanding through the use of plot devices (e.g. back story and cut scenes). The gender considers the different perspectives between male and female players. In order to be genderinclusive, the DLG conflict design should include appropriate role models. The social collaboration concerns the social practice side of learning. An educational game should harness dialog, altruism, reciprocity, collective action and solidarity to support the development of a community of peers [4]. The challenges-puzzlesquests are the core of the learning activities. Well designed challenges generate tacit knowledge through knowledge exposition, conversations and reflection.

GOM-II is an evolution of the GOM framework, successfully used to design the academic adventure game Zadahr [12]. GOM-II was developed based on insights acquired on the design of the educational adventure game $\gamma$ Khozi $[4$, 13]. However, no game so far is reported to use GOM-II in its design.

\section{$5 \quad$ Three Layered Thinking Model}

The Three Layered Thinking Model (TLT) aims at supporting the design of web-based educational games, and stresses the importance of decreasing task complexity to adapt to limited budget environments. The model is structured 
in three levels, in which the pedagogic level (knowledge production) and the achievement level (knowledge outcomes) influence the core design level [6].

The pedagogic level relates to the knowledge production targeted by the DLG. On this level, the designer has to transform curriculum into game goals, considering the previous skills of the player and the desired knowledge enhancement. The achievement level relates to the knowledge outcomes of the DLG. A designer needs to conceive a DLG aiming to achieve a higher knowledge production [6].

The design level is the core level, aimed to guarantee the requirements of the pedagogic level and to achieve the motivators of the achievement level. It includes designing the style, task and interface of the DLG. The style design of the DLG, i.e. the definition of game genre, number of players, camera style, etc, should match the game goals (defined on the pedagogic level). The task design of the DLG, i.e. the design of levels, challenges and puzzles, should enhance players knowledge and skills through challenge provision. The tasks should consider the player previous skills and the desired knowledge enhancement (defined on pedagogic level) and also provide pleasure and challenge (motivators of achievement level) to the player. The game interface design should help the player keep concentrated in the game (another motivator of the achievement level) [6].

The authors carried out the design and development of three games to empirically test the Three Layered Thinking Model. The games were applied on a group of 120 undergraduate students of a web-based course on introduction to software applications. The authors tested different game styles, tasks and interfaces to achieve different curriculum goals. They conducted surveys to assess the motivation of the games, isolating and weighting the four motivators (skill, challenge, concentration and pleasure). They also used log data to assess the time spent on the game and how frequently the students played the game. Results indicated that the produced games do encourage learners engagement [6].

\section{Educational Games Design Framework}

The Educational Game Design Framework (EGDF) is focused on producing games for higher education, in which students need to self-learn specific subjects or materials, with integrated self-assessment modules. The model combines three main factors: game design, pedagogy and learning content modeling [5].

In game design, the focus is on usability and multi-modality. The DLG design should consider the usability test items of effectiveness, efficiency and satisfaction, based on ISO 9241 [15] and on the heuristics of Pinelle [16]. The multimodality component uses the multimedia aspect of the game to provide fun and engage. In this aspect, the authors suggest the use of the heuristics of Malone [17] to generate challenge, fantasy, and curiosity in the game.

The pedagogy ensures that the DLG meets the learning outcomes. The authors suggest that the DLG subject selection should consider Bloom's taxonomy of learning outcomes [18] and motivation theory [19] to evaluate how the game affects students' motivation. The pedagogy should lead to appropriate learning content modeling, providing verifiable learning outcomes, in order to guarantee 
the achievement of learning goals. No game developed with this framework was found in the literature.

\section{Comparison}

The above mentioned frameworks aim at establishing patterns and guidelines for effective DLGs design. A comparison among them clarifies the underlying common characteristics and distinct aspects. Such a comparison has a twofold purpose: first it serves as a initial step for a DLG designer interested to adopt a methodological framework, and secondly unveils open problems and opportunities for further research and experimentation.

\subsection{The pedagogical base}

To some extent, all analyzed frameworks cite some learning theory that pedagogically justifies their development. Table 1 relates the frameworks with their pedagogical bases, which are briefly recalled in the rest of this Section.

Table 1. Learning theories used in the frameworks

\begin{tabular}{l|l}
\hline Framework & Pedagogical Base \\
\hline Retain & Blooms taxonomy [18], Piaget's schemes [22] and Gagne's events [23] \\
\hline 'I's & Vygotsky ZPD [20] and Piaget's schemes [22] \\
\hline GOM - II & Vygotsky ZPD [20] \\
\hline TLT & Blooms taxonomy [18] and Siang hierarchal learning theories [21] \\
\hline EGDF & Blooms taxonomy [18] \\
\hline
\end{tabular}

Bloom's taxonomy of educational objectives states that targeted academic content needs to be introduced and reused in a hierarchical manner [18]. This is the most popular pedagogical base, being used in three of the analyzed frameworks (RETAIN, EGDF and Three Layered Thinking). This is due to the close alignment of Bloom's main argument with the digital gaming activity itself. In most games, to advance to the next level, the player is required to learn the rules of gameplay (and thus the associated learning objects) and how to apply them on the present level.

Piaget's schemes relate learning with adaptation, which is composed of assimilation and accommodation. Assimilation is the process of understanding world through existing schemes, whereas accommodation is the process of building new schemes [22]. RETAIN and 'I's frameworks state the importance of adapting the increasing complexity of challenges in a DLG to provide assimilation and accommodation to the player.

Vygotsky zones of proximal development (ZPD) focus on the difference between a child's actual and potential levels of development [20]. ZPD is the pedagogical base of two frameworks (GOM-II and 'I's). A well-designed DLG acts as a mentor, to move players from their actual to their potential development level [4].

The selection of a learning theory depends on what needs to be taught, how it is to be taught, and to whom it is being taught [7]. Therefore, the knowledge 
about the underlying pedagogical base is important for the designer to choose the methodological framework adequate to his case.

\subsection{Interactivity, engagement and progression of challenges}

Two other elements are common to all analyzed frameworks - interactivity and engagement; and increasing complexity of challenges.

Interactivity and engagement are fundamental to immerse the player. A fully immersed player intellectually invests in the context of a game - which is ideal for the learning situation [7]. Interactivity and engagement are fundamental factors to immerse a player. Interactivity should be approached through appropriate feedback and user interface [8], possibly grounded on usability [16] and ergonomic design $[5,15]$. Engagement should be promoted by adequate narrative [4] and challenges design [6]. The heuristics of Malone [5, 17]) could be considered to provide higher engagement. Specifically about immersion, three frameworks (GOM-II, Three Layered Thinking and 'I's) cite the concept of flow, linking four factors - skill, challenge, concentration and pleasure - to the achievement of the state in which people are so involved in an activity that nothing else seems to matter[14].

Increasing complexity of challenges is fundamental to keep the player immersed. Increasing complexity of challenges is a common feature to all kinds of digital games. In DLGs it is specially important, considering that challenges should promote both pleasure and learning. Adequate challenges increasing complexity should consider player previous skills [6] and previous learned materials [7], aiming at a pleasurable frustration state, in which the player feels stimulated to try harder when facing a defeat [8].

\subsection{Targets, DLG design, assessment and experimental validation}

Table 2 compares the analyzed frameworks in terms of their target, the presence of practical game design guidelines, the availability of assessment data of DLGs and the frameworks experimental validation.

Table 2. Frameworks comparison table

\begin{tabular}{l|l|l|l|l}
\hline Framework & Target & $\begin{array}{l}\text { Practical } \\
\text { guidelines }\end{array}$ & $\begin{array}{l}\text { DLG assess- } \\
\text { ment }\end{array}$ & $\begin{array}{l}\text { Experimental vali- } \\
\text { dation }\end{array}$ \\
\hline RETAIN & Generalist & No & Yes & N/A \\
\hline I's & Generalist & No & No & (game concept) \\
\hline GOM - II & Specialist & No & Yes & N/A \\
\hline TLT & Specialist & No & No & Empirically tested \\
\hline EGDF & Specialist & Yes & No & N/A \\
\hline
\end{tabular}

The analyzed frameworks can be classified according to the target as generalists or specialists. The generalist RETAIN and 'I's frameworks can be used to design any kind of DLG to any target audience. On the other hand, the specialist frameworks focus on a platform (web-based games in three layered thinking framework), a target audience (higher education students in Educational Game 
Design Framework) or a game genre (adventure games in GOM II). Specialist frameworks offer specific guidelines to their target (i.e. platform, target audience or game genre). Regarding practical guidelines to support the DLG design, EGDF suggests the utilization of heuristics $[16,17]$ to provide usability and to engage the player. The other four frameworks are limited to high-level methodological guidelines and do not provide procedural guidance to structure the development process of a DLG. It is also possible to use two frameworks, RETAIN and GOM-II, to assess already developed DLGs. The first uses a score from 0 to 63 , in which the higher the score, the better the designed DLG, while the second proposes the use of a checklist to control the presence of all the necessary criteria established by the framework. Finally, about experimental validation, only the Three Layered Thinking framework was validated in a field study. The DLGs were developed for the same team that created the framework, and evaluated with a group of undergraduate students. The 'I's framework presents a game concept, developed according the framework guidelines. However, no study was found detailing the actual implementation. All the other frameworks consider the experimental validation as a future work $[4,5,7,8]$.

\section{Open problems and prominent research issues}

The methodological frameworks are still lacking a thorough and independent assessment. Although based on previous research and strong instructional theories, few DLGs were designed and no updates for the analyzed frameworks were encountered in recent years. Moreover, all the developed DLGs were published by the same research teams that developed the frameworks. These factors indicate a fragility of the active community of users of the frameworks, specially if considered the robust amount of DLGs developed and published in recent years. Although GOM-II and Three Layered Thinking are more mature in relation to the other frameworks, no game was actually designed with GOM-II and the empirical testing of Three Layered Thinking was carried out by the same research team that proposed the framework. To overcome this issue, further research should focus on the design of framework-based DLGs. Only through empiric use, the frameworks would be adequately assessed (and possibly updated) and a community of users can appear.

Another issue is the absence of information about how the frameworks principles can be applied to game design in practice. Framework-based designed DLGs would also help to solve this issue and would permit further investigations, for example: (i) how to map learning goals to game goals; and (ii) how to test the effectiveness of design practices suggested by the frameworks. In addition, with diverse framework-based designed DLGs, it would possible to establish comparisons among them, investigating, for example, how the guidelines on generalist frameworks correlate with different game mechanics. Finally, the DLGs should preferably be designed by different research teams from the ones that developed the framework. This would testify about the real applicability of the frameworks, meanwhile a bigger community of users is more likely to bring useful insights for frameworks updates. 


\section{Conclusions and future work}

The comparison (i) indicates that interactivity, engagement and increasing complexity of challenges are fundamental factors to DLGs design; and (ii) establishes pedagogical base, target, presence of practical guidelines and possibility of game assessment as selecting criteria to guide the choice for a framework. This paper detected two main research issues: (i) the lack of independent assessment, and (ii) the uncertainty about how the principles can be applied to game design in practice. Both issues should be approached through design and development preferably by different research teams - of framework-based DLGs. This would provide important empirical data to the field, validating the frameworks, stimulating their popularization, and enabling further scientific investigation.

This research is a likely subject to further extension. Due to size constraints of this paper, and giving preference for more extensively detailed works, some frameworks [24-28] were not included in this research. In addition, during the review of this paper, the works from [29] and [30] detailed new methodological frameworks that need future analysis. The extension of this comparison study can strength the achieved conclusions and harness the identification of gaps in the state of the art.

\section{References}

1. Connolly, T. M., Boyle, E. A., MacArthur, E., Hainey, T., \& Boyle, J. M.: A systematic literature review of empirical evidence on computer games and serious games. Computers \& Education 59(2), 661-686 (2012)

2. Susi, T., Johannesson, M., Backlund, P.: Serious games: An overview. IKI technical reports, (2007)

3. Kebritchi, M.: Examining the pedagogical foundations of modern educational computer games, Computers \& Education 51(4), 1729-1743 (2008)

4. Amory, A.: Game object model version II: a theoretical framework for educational game development. Educational Technology Research and Development 55(1), 5177 (2007)

5. Ibrahim, R., Azizah J.: Educational Games (EG) design framework: Combination of game design, pedagogy and content modeling. In: International Conference on Electrical Engineering and Informatics, pp. 293-298. IEEE Press, New York (2009)

6. Fu, F., Yu S.: Three layered thinking model for designing web-based educational games. In: Advances in Web Based Learning-ICWL, pp. 265-274. Springer Berlin Heidelberg (2008)

7. Gunter, G. A., Kenny, R. F., Vick, E.H.: Taking educational games seriously: using the retain model to design endogenous fantasy into standalone educational games. Educational Technology Research and Development 56(5-6), 511-537 (2008)

8. Annetta, L. A. . The I's have it: A framework for serious educational game design. Review of General Psychology 14(2), 105-112 (2010)

9. Catalano, C. E., Luccini, A. M., Mortara, M.: Guidelines for an effective design of serious games. International Journal of Serious Games 1(1), (2014)

10. Gloria, A. de., Bellotti, F., Berta, R.: Serious games for education and training, International Journal of Serious Games, 1(1) (2014) 
11. Dondlinger, M. J.: Educational video game design: A review of the literature. Journal of applied educational technology 4(1), 21-31 (2007)

12. Amory, A.: Building an educational adventure game: theory, design, and lessons. Journal of Interactive Learning Research 12(2), 249-263 (2001)

13. Amory, A.: Learning to play games or playing games to learn? A health education case study with Soweto teenagers. Australasian Journal of Educational Technology 26(6), 810-829 (2010)

14. Csikszentmihalyi, M., Csikzentmihaly, M.: Flow: The psychology of optimal experience. Harper Perennial, New York (1991)

15. Standard, I. "Ergonomic requirements for office work with visual display terminals (vdts)part 11: Guidance on usability" International Organization for Standardization (1998)

16. Pinelle, D., Wong, N., Stach, T.: Heuristic evaluation for games: usability principles for video game design. In: Proceedings of the SIGCHI Conference on Human Factors in Computing Systems, pp. 1453-1462. ACM Press, New York (2008)

17. Malone, T. W.: What makes things fun to learn? Heuristics for designing instructional computer games. In: Proceedings of the 3rd ACM SIGSMALL symposium and the first SIGPC symposium on Small systems, pp. 162-169. ACM Press, New York (1980)

18. Bloom, B. S.: Taxonomy of educational objectives: The classification of education goals by a committee of college and university examiners. David McKay, (1956)

19. Kanfer, R.: Motivation theory and industrial and organizational psychology. Handbook of industrial and organizational psychology 1(2), 75-130 (1990)

20. Vygotsky, L. S.: Mind in society: The development of higher mental process. Harvard University Press, Cambridge (1978)

21. Siang, A. C., Rao, R. K.: Theories of learning: a computer game perspective. In: Fifth International Symposium on Multimedia Software Engineering, pp. 239-245. IEEE Press, New York (2003)

22. Piaget, J.: The origins of intelligence in children. International Universities Press, New York (1952)

23. Gagne, R. M.: Instructional technology: foundations. Routledge, New York (2013)

24. Kiili, K.: Digital game-based learning: Towards an experiential gaming model. The Internet and higher education 8(1), 13-24 (2005)

25. Tan, P. H., Ling, S. W., Ting, C. Y.: Adaptive digital game-based learning framework. In: 2nd international conference on Digital interactive media in entertainment and arts, pp. 142-146. ACM Press, New York, (2007)

26. Song, M., Zhang, S.: EFM: A model for educational game design. In Technologies for e-learning and digital entertainment, pp. 509-517. Springer Berlin Heidelberg, (2008)

27. Yusoff, A., Crowder, R., Gilbert, L., Wills, G.: A conceptual framework for serious games. In Ninth IEEE International Conference on Advanced Learning Technologies, pp. 21-23. IEEE Press, New York (2009)

28. Mitgutsch, K., Alvarado, N.: Purposeful by design?: a serious game design assessment framework. In: Proceedings of the International Conference on the foundations of digital games, pp. 121-128. ACM Press, New York (2012)

29. Arnab, S., Lim, T., Carvalho, M. B., Bellotti, F., Freitas, S., Louchart, S., Suttie, N., Berta, R., De Gloria, A.: Mapping learning and game mechanics for serious games analysis. British Journal of Educational Technology 46(2), 391-411 (2015)

30. Carvalho, M. B., Bellotti, F., Berta, R., De Gloria, A., Sedano, C. I., Hauge, J. B., Hu, J., Rauterberg, M.: An activity theory-based model for serious games analysis and conceptual design. Computers \& Education 87, 166-181 (2015) 